\title{
On OTFS Modulation for High-Doppler Fading Channels
}

\author{
K. R. Murali and A. Chockalingam \\ Department of ECE, Indian Institute of Science, Bangalore 560012, India
}

\begin{abstract}
Orthogonal time frequency space (OTFS) modulation is a 2-dimensional (2D) modulation scheme designed in the delay-Doppler domain, unlike traditional modulation schemes which are designed in the time-frequency domain. Through a series of 2D transformations, OTFS converts a doubly-dispersive channel into an almost non-fading channel in the delay-Doppler domain. In this domain, each symbol in a frame experiences an almost constant fade, thus achieving significant performance gains over existing modulation schemes such as OFDM. The sparse delay-Doppler impulse response which reflects the actual physical geometry of the wireless channel enables efficient channel estimation, especially in high-Doppler fading channels. This paper investigates OTFS from a signal detection and channel estimation perspective, and proposes a Markov chain MonteCarlo sampling based detection scheme and a pseudo-random noise (PN) pilot based channel estimation scheme in the delayDoppler domain.
\end{abstract}

keywords: OTFS modulation, $2 D$ modulation, delay-Doppler domain, OTFS signal detection, Markov chain Monte Carlo sampling, delay-Doppler channel estimation.

\section{INTRODUCTION}

| Mobile radio channels are doubly-dispersive channels, where multipath propagation effects cause time dispersion and Doppler shifts cause frequency dispersion [1]. Multicarrier signaling schemes such as OFDM are often employed to alleviate the effect of inter-symbol interference (ISI) caused by time dispersion [2]. Doppler shifts result in inter-carrier interference (ICI) in OFDM which degrades performance [3]. An approach to combat ISI and ICI in OFDM is pulse shaping. Pulse shaped OFDM systems use general time-frequency lattices and optimized pulse shapes in the time-frequency domain [4]-[6]. The resilience against time-frequency dispersions in an OFDM system depends on the time-frequency localization of the pulse (due to Heisenberg's uncertainty principle) and the distance between lattice points in the time-frequency (TF) plane [4]. Using results from sphere packing theory, [4] shows how to optimally design lattice and pulse shape for lattice-OFDM (LOFDM) systems in doubly-dispersive channels to jointly minimize the ISI/ICI. A transmission scheme employing overcomplete Weyl-Heisenberg (W-H) frames as modulation pulses is proposed in [5]. In [6], the lattice parameters and pulse shape of the modulation waveform are jointly optimized to adapt to the channel scattering function from the viewpoint of minimum symbol energy perturbation. While the LOFDM system in [4] confines the transmission pulses to a set of orthogonal basis functions, the pulse design in [5],[6] relaxed this orthogonality constraint in order to improve the TF concentration of the initial pulses, and in the process making the

This work was supported in part by the J. C. Bose National Fellowship, Department of Science and Technology, Government of India. system more susceptible to ISI. However, these and other such systems that employ the pulse shaping approach are inadequate to efficiently address the need to support high Doppler shifts expected in future wireless systems including $5 \mathrm{G}$ systems, where operation in high mobility scenarios (e.g., high-speed trains) and operation in millimeter wave (mmWave) bands are envisioned [7]. Orthogonal time frequency space (OTFS) modulation, a recently proposed modulation scheme [7]-[9], has attractive signaling attributes that can meet the high-Doppler signaling need through a different approach, namely, signaling in the delay-Doppler domain (instead of the conventional approach of signaling in the time-frequency domain). In this paper, we investigate OTFS with emphasis on low-complexity OTFS signal detection and channel estimation in the delayDoppler domain.

OTFS waveform, having its origin from representation theory, is a waveform resilient to delay-Doppler shifts in the wireless channel [7]-[9]. The idea is to transform the timevarying multipath channel into a $2 \mathrm{D}$ channel in the delayDoppler domain and to carry out modulation and demodulation in this domain. Due to Heisenberg's uncertainty principle, a signal cannot be localized both in time and frequency simultaneously. But OTFS waveform is localized in the delayDoppler domain, and TDMA and OFDM become limiting cases of OTFS when viewed in this domain. In OTFS, 2D basis functions that are delocalized in the time-frequency plane but are localized in the delay-Doppler plane are used. Information symbols are mapped onto these 2D basis functions that span the bandwidth and time duration of the transmission frame. This transformation along with equalization in this domain makes all the symbols over a transmission frame experience the same channel gain, leading to good performance in highDoppler channels. OTFS specializes to CDMA and OFDM if 1D basis functions (spreading codes and subcarriers, respectively) are used in place of 2D basis functions. Thus OTFS has all the advantages of TDMA, OFDM, and CDMA, and it can be viewed as the mother waveform of the above three. Another interesting aspect of OTFS from an implementation view-point is that it can be realized by adding pre- and postprocessing blocks to filtered OFDM systems.

OTFS has been shown to exhibit significantly lower block error rates compared to OFDM over a wide range of Doppler shifts (for vehicle speeds ranging from $30 \mathrm{~km} / \mathrm{h}$ to $500 \mathrm{~km} / \mathrm{h}$ in $4 \mathrm{GHz}$ band), and that the robustness to high-Doppler channels (500 km/h vehicle speeds) is especially notable, as OFDM performance breaks down in such high-Doppler scenarios [8]. Also, OTFS has been shown to offer bit error rate (BER) performance advantage compared to OFDM in mmWave systems (28 GHz band) which encounter high frequency dispersion due 


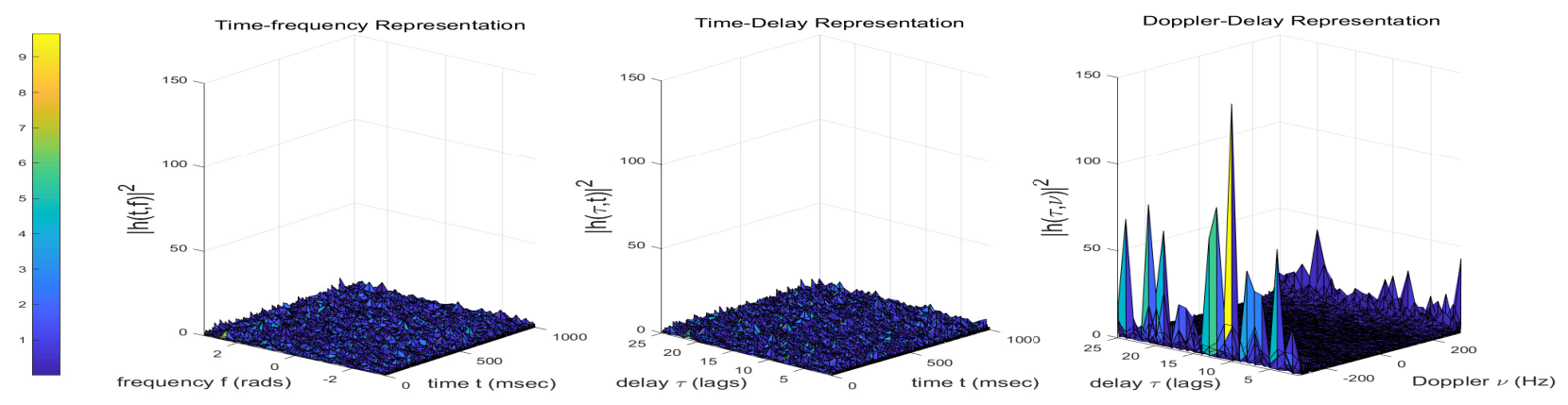

Fig. 1. Squared magnitude of the impulse response of a $300 \mathrm{~Hz}$ Jakes Doppler channel model with 25 uniform power delay profile taps in (a) time-frequency domain, (b) time-delay domain, and (c) Doppler-delay domain.

to phase noise and high Dopplers [9]. An equivalent channel matrix representation and a two-stage equalizer for OTFS are presented in [10]. Vectorized formulations of the inputoutput relation describing OTFS modulation and demodulation are presented in [11],[12]. A message passing based OTFS signal detection scheme based on the vectorized formulation is presented in [11]. MIMO OFDM-based OTFS and its vectorized formulation that can enable MIMO OTFS analysis and implementation are presented in [13]. Recognizing that the description of the OTFS waveform design framework can admit multiple waveforms with differences in performance depending on the delay spread and Doppler spread of the channel, [14] presents another modulation scheme which is robust in high-Doppler and low delay spread channels, termed as frequency-domain multiplexing with frequency-domain cyclic prefix (FDM-FDCP).

Our contribution in this paper adds to the OTFS literature that has been building up recently. Our contributions are two-fold. First, leveraging the vectorized formulations and assuming perfect knowledge of the equivalent channel matrix, we propose a Markov chain Monte Carlo (MCMC) sampling based low-complexity OTFS signal detection scheme. Second, we relax the perfect knowledge of the equivalent channel matrix and present a pseudo-noise (PN) sequence pilot based channel estimation scheme in the delay-Doppler domain. In this context, we note that the detection performance of OTFS reported in the literature (e.g., [7],[11]) assume perfect channel knowledge.

The rest of this paper is organized as follows. The delayDoppler channel representation and characteristics are presented in Sec. III The OTFS modulation and the linear vector system model are introduced in Sec. III OTFS signal detection using MCMC sampling techniques and the resulting bit error performance in high-Doppler scenarios are presented in Sec. IV Channel estimation in the delay-Doppler domain and OTFS performance with estimated channel are presented in Sec. V Conclusions are presented in Sec. VI.

\section{Wireless Channel in Delay-Doppler Domain}

When a waveform is transmitted, the wireless channel delays it in time (delay shift) and shifts its frequency contents
(Doppler shift), and a delay-Doppler shifted waveform is received. A linear time-varying multipath channel can be represented in different ways, depending upon the parameters used for modeling the impulse response, namely, time-frequency, time-delay, Doppler-delay. Usually, time-delay representation $h(t, \tau)$ or, equivalently, time-frequency representation $H(t, f)$ are used, where $t, \tau$, and $f$ denote time, delay, and frequency, respectively. These representations have a finite support characterized by the maximum delay and Doppler spreads. The rate at which the channel coefficients vary $(\propto 1 /$ coherence time $)$ in these representations depends on the mobility and operating frequency. High mobility or high operating frequency would cause the channel to vary rapidly, making channel estimation and the associated time-frequency adaptation difficult.

An equivalent compact way of representing the channel is to use delay-Doppler impulse response $h(\tau, \nu)$, where $\tau$ and $\nu$ denote the delay and Doppler, respectively [7],[8]. The taps in this domain correspond to the group of reflectors having a particular delay (depends on reflectors' relative distance) and Doppler value (depends on reflectors' relative velocity). Thus, this representation reflects the actual geometry of the wireless channel [7]. Since there are only a small number of group of reflectors with different delay and Doppler values [8], the parameters that need to be estimated are also fewer, and the representation in this domain is more compact and sparse. Also, the velocity and distance remain roughly the same for at least few milliseconds, and thus the delay-Doppler taps are time invariant for a larger observation time as compared to that in time-frequency representation [7]. This makes channel estimation easy in the delay-Doppler domain.

As an illustration, we have plotted the squared magnitude of the impulse response of a $300 \mathrm{~Hz}$ (maximum Doppler) Jakes channel model [1] with 25 delay taps and uniform power delay profile in Fig. 1. (a) in time-frequency domain, (b) in timedelay domain which has a Fourier transform relation with the time-frequency domain along the delay axis, and (c) in delayDoppler domain which is related to the time-frequency domain by a transform called 2D symplectic Fourier transform [7]. As we can see from Fig. 11 the impulse response is not localized in the time-frequency and time-delay representations, whereas it is peaky (localized) in a few delay-Doppler bins in the delay- 


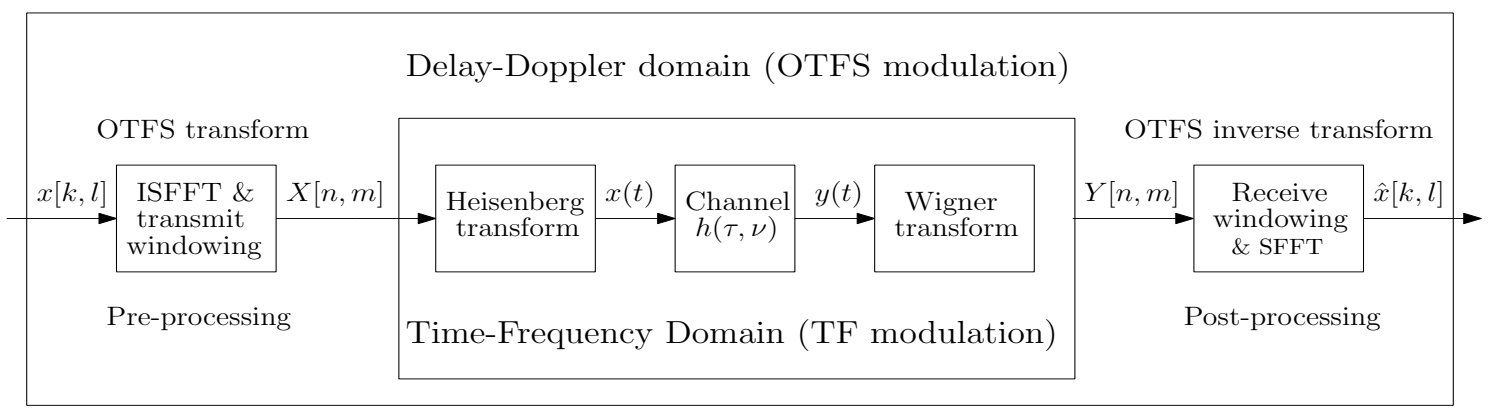

Fig. 2. Block diagram of OTFS modulation scheme.

Doppler representation, i.e., the impulse response is sparse in the delay-Doppler representation. This characteristic can be exploited for efficient channel estimation, as we will see in Sec. V]

In the delay-Doppler representation, the received signal $y(t)$ is the sum of reflected copies of the transmitted signal $x(t)$ which are delayed in time $(\tau)$ and shifted in frequency $(\nu)$ by the reflectors [7]. Thus, the coupling between an input signal and the channel in this domain is given by the following double integral:

$$
y(t)=\int_{\nu} \int_{\tau} h(\tau, \nu) x(t-\tau) e^{j 2 \pi \nu(t-\tau)} \mathrm{d} \tau \mathrm{d} \nu .
$$

While channel representation is one use of the delay-Doppler domain, information carrying symbols themselves can reside in this domain. OTFS modulation is based on this [7],[8].

\section{OTFS MODULATION}

OTFS modulation, when implemented using pre- and postprocessing to existing multicarrier modulation schemes, can be viewed as a series of transformations at the transmitter and receiver. The block diagram of the OTFS modulation scheme is shown in Fig. 2, where the inner box is the familiar multicarrier (TF) modulation, and the outer box with a preand post-processor implements the OTFS modulation scheme in the delay-Doppler domain.

The information symbols $x[k, l]$ (e.g., QAM symbols) residing in the delay-Doppler domain are first mapped to the familiar time-frequency domain symbols $X[n, m]$ through a transform called the 2D inverse symplectic finite Fourier transform (ISFFT) and windowing, together called the OTFS transform. Each $x[k, l]$ modulates a $2 \mathrm{D}$ basis function that completely spans the transmission time and bandwidth in the TF domain. The Heisenberg transform, which is a generalization of the OFDM transform, is then applied to the time-frequency transformed symbols $X[n, m]$ to convert to the time domain signal $x(t)$ for transmission. At the receiver, the received signal $y(t)$ is transformed back to a time-frequency domain signal $Y[n, m]$ through Wigner transform (inverse of the Heisenberg transform), which is a generalization of the inverse OFDM transform. Subsequently, $Y[n, m]$ is transformed to the delayDoppler domain signal $y[k, l]$ through the symplectic finite Fourier transform (SFFT) for demodulation. As we can see, OTFS modulation can be viewed as a scheme with additional pre- and post-processing to a multicarrier system that uses TF modulation. In the following subsections, we describe the signal models in TF modulation and OTFS modulation, and present a discrete linear vector channel model of the received OTFS signal.

\section{A. Time-frequency modulation and the TF lattice}

- Definitions/notation:

- A lattice in the TF plane is a sampling of the time axis at an interval $T$ and the frequency axis at an interval $\Delta f$ denoted by

$$
L=\{(n T, m \Delta f), n=0, \cdots, N-1, m=0, \cdots, M-1\} .
$$

- A packet burst occupies $N T$ seconds in time and $M \Delta f \mathrm{~Hz}$ in bandwidth.

- Information symbols $X[n, m], n=0, \cdots, N-1$, $m=0, \cdots, M-1$ are transmitted in a given packet burst.

- Transmit and receive pulses $\varphi_{t x}(t)$ and $\varphi_{r x}(t)$, respectively, which are bi-orthogonal with respect to time and frequency translations are used for modulation and demodulation, which eliminates cross symbol interference.

In TF modulation, the symbols $X[n, m]$ in $\mathrm{TF}$ lattice are transmitted using the translations and modulations of the transmit pulse $\varphi_{t x}(t)$ as the basis functions as follows:

$$
x(t)=\sum_{n=0}^{N-1} \sum_{m=0}^{M-1} X[n, m] \varphi_{t x}(t-n T) e^{j 2 \pi m \Delta f(t-n T)} .
$$

This can be interpreted as a linear operator called the Heisenberg operator parametrized by $X[n, m]$ and operating on $\varphi_{t x}(t)$. The transmitted signal $x(t)$ is received as $y(t)$ given by (1), which can also be viewed as another Heisenberg operator parametrized by $h(\tau, \nu)$ and operating on $x(t)$. Thus, the cascaded Heisenberg operator property can be used in the derivation of input-output relationship as in [8].

In TF demodulation, the channel distorted signal $y(t)$ is matched filtered with the receive pulse $\varphi_{r x}(t)$ to obtain the sufficient statistic for detection. This is done in 2 steps. First, the cross ambiguity function is calculated as follows:

$$
A_{\varphi_{r x}, y}(\tau, \nu)=\int \varphi_{r x}^{*}(t-\tau) y(t) e^{-j 2 \pi \nu(t-\tau)} \mathrm{d} t .
$$


This is then sampled at an interval $\tau=n T$ and $\nu=m \Delta f$ to get the matched filter output, given by

$$
Y[n, m]=\left.A_{\varphi_{r x}, y}(\tau, \nu)\right|_{\tau=n T, \nu=m \Delta f},
$$

where (4) is called the Wigner transform.

Suppose the noise is additive white Gaussian, denoted by $v(t)$, and the impulse response $h(\tau, \nu)$ has finite support bounded by $\left(\tau_{\max }, \nu_{\max }\right)$. If $A_{\varphi_{r x}, y}(\tau, \nu)=0$ for $\tau \in$ $\left(n T-\tau_{\max }, n T+\tau_{\max }\right), \nu \in\left(m \Delta f-\nu_{\max }, m \Delta f+\nu_{\max }\right)$, then the relation between $Y[n, m]$ and $X[n, m]$ can be derived as [8]

$$
Y[n, m]=H[n, m] X[n, m]+V[n, m],
$$

where $V[n, m]=\left.A_{\varphi_{r x}, v}(\tau, \nu)\right|_{\tau=n T, \nu=m \Delta f}$ and $H[n, m]$ is given by

$$
H[n, m]=\int_{\tau} \int_{\nu} h(\tau, \nu) e^{j 2 \pi \nu n T} e^{-j 2 \pi(\nu+m \Delta f) \tau} \mathrm{d} \nu \mathrm{d} \tau .
$$

Clearly, each symbol $X[n, m]$ in a frame (packet burst) gets multiplied by a different fade $H[n, m]$ in TF modulation. However, each symbol in the delay-Doppler domain will be multiplied by an almost constant fade in the OTFS modulation presented in the following subsection.

\section{B. OTFS modulation and the delay-Doppler lattice}

The delay-Doppler signal representation is a quasi periodic representation, with a delay period, $\tau_{r}=\frac{1}{\Delta f}$ and a Doppler period, $\nu_{r}=\frac{1}{T}$, such that $\tau_{r} \nu_{r}=1$. Thus, the delay-Doppler lattice given below can be considered as the sampling of delay and Doppler axes within a rectangle of unit area $\left(\tau_{r} \nu_{r}=1\right)$ in the delay-Doppler domain. The delay-Doppler representation is non-unique because $\tau_{r}$ and $\nu_{r}$ can take any value, such that $\tau_{r} \nu_{r}=1$. In the limit $\tau_{r}$ tending to infinity and $\nu_{r}$ tending to zero, the delay-Doppler representation becomes the familiar temporal representation. Similarly, in the limit $\nu_{r}$ tending to infinity and $\tau_{r}$ tending to zero, the delayDoppler representation becomes the familiar frequency domain representation of the signal.

- Definitions/notation:

- A lattice in the delay-Doppler plane is a sampling of the delay axis at an interval $\frac{1}{M \Delta f}$ and the Doppler axis at an interval $\frac{1}{N T}$, denoted by

$$
L_{d D}=\left\{\left(\frac{k}{N T}, \frac{l}{M \Delta f}\right), k=0, \cdots, N-1, l=0, \cdots, M-1\right\} .
$$

- Let $X_{p}[n, m]$ be the periodized version of $X[n, m]$ with period $(N, M)$. The SFFT of $X_{p}[n, m]$ is then given by

$$
x_{p}[k, l]=\sum_{n=0}^{N-1} \sum_{m=0}^{M-1} X_{p}[n, m] e^{-j 2 \pi\left(\frac{n k}{N}-\frac{m l}{M}\right)},
$$

and the ISFFT is $X_{p}[n, m]=\operatorname{SFFT}^{-1}(x[k, l])$, given by

$$
X_{p}[n, m]=\frac{1}{M N} \sum_{k=0}^{N-1} \sum_{l=0}^{M-1} x[k, l] e^{j 2 \pi\left(\frac{n k}{N}-\frac{m l}{M}\right)} .
$$

In OTFS modulation, the information symbols in the delayDoppler domain $x[k, l]$ are mapped to TF domain symbols $X[n, m]$ as

$$
X[n, m]=W_{t x}[n, m] S F F T^{-1}(x[k, l]),
$$

where $W_{t x}[n, m]$ is the transmit windowing square summable function. $X[n, m]$ thus obtained is in the TF domain and it is TF modulated as described in [II-A and $Y[n, m]$ is obtained by (3) and (4).

In OTFS demodulation, a receive window $W_{r x}[n, m]$ is applied to $Y[n, m]$ and periodized to obtain $Y_{p}[n, m]$ which has the period $(N, M)$, as

$$
\begin{aligned}
Y_{W}[n, m] & =W_{r x}[n, m] Y[n, m], \\
Y_{p}[n, m] & =\sum_{k, l=-\infty}^{\infty} Y_{W}[n-k N, m-l M] .
\end{aligned}
$$

The symplectic Fourier transform is then applied to $Y_{p}[n, m]$ to convert it from TF domain back to delay-Doppler domain $\hat{x}[k, l]$, as

$$
\hat{x}[k, l]=\operatorname{SFFT}\left(Y_{p}[n, m]\right) .
$$

Therefore, the input-output relation in OTFS modulation can be derived as [8]

$$
\hat{x}[k, l]=\frac{1}{M N} \sum_{m=0}^{M-1} \sum_{n=0}^{N-1} x[n, m] h_{w}\left(\frac{k-n}{N T}, \frac{l-m}{M \Delta f}\right),
$$

where

$$
h_{w}\left(\frac{k-n}{N T}, \frac{l-m}{M \Delta f}\right)=\left.h_{w}\left(\nu^{\prime}, \tau^{\prime}\right)\right|_{\nu^{\prime}=\frac{k-n}{N T}, \tau^{\prime}=\frac{l-m}{M \Delta f}},
$$

where $h_{w}\left(\nu^{\prime}, \tau^{\prime}\right)$ is the circular convolution of the channel response with a windowing function $w(\tau, \nu)$, given by

$$
h_{w}\left(\nu^{\prime}, \tau^{\prime}\right)=\int_{\nu} \int_{\tau} h(\tau, \nu) w\left(\nu^{\prime}-\nu, \tau^{\prime}-\tau\right) \mathrm{d} \tau \mathrm{d} \nu .
$$

The windowing function $w(\tau, \nu)$ is the symplectic discrete Fourier transform (SDFT) of the time frequency window $W[n, m]=W_{t x}[n, m] W_{r x}[n, m]$, i.e.,

$$
w(\tau, \nu)=\sum_{m=0}^{M-1} \sum_{n=0}^{N-1} W[n, m] e^{-j 2 \pi(\nu n T-\tau m \Delta f)} .
$$

From (10), we see that each demodulated symbol $\hat{x}[k, l]$ for a given value of $k$ and $l$ experiences the same fade $h_{w}(0,0)$ on the transmitted symbol $x[k, l]$ and the cross symbol interference vanishes if

$$
h_{w}\left(\frac{k-n}{N T}, \frac{l-m}{M \Delta f}\right) \approx 0 \forall n \neq k, m \neq l .
$$

This condition depends on the delay and Doppler spreads of the channel and the windows used in the modulation. Thus, each symbol in a given frame experiences an almost constant fade $h_{w}(0,0)$. 


\section{Vectorized formulation of the input-output relation}

Assume that there are $P$ taps (signal propagation paths). The parameter $P$ is also called the sparsity of the channel. Let the path $i$ be associated with a delay $\tau_{i}$, Doppler $\nu_{i}$, and a fade coefficient $h_{i}$. The impulse response in the delay-Doppler domain can be written as

$$
h(\tau, \nu)=\sum_{i=1}^{P} h_{i} \delta\left(\tau-\tau_{i}\right) \delta\left(\nu-\nu_{i}\right)
$$

Assuming the windows used in modulation $\left(W_{t x}[n, m]\right)$ and demodulation $\left(W_{r x}[n, m]\right)$ to be rectangular, the input-output relation in (10) for the above channel can be derived as [11]

$$
\begin{aligned}
& h_{w}\left(\tau^{\prime}, \nu^{\prime}\right)=\sum_{i=1}^{P} h_{i} e^{-j 2 \pi \nu_{i} \tau_{i}} w\left(\nu^{\prime}-\nu_{i}, \tau^{\prime}-\tau_{i}\right) \\
& =\sum_{i=1}^{P} h_{i} e^{-j 2 \pi \nu_{i} \tau_{i}} \sum_{c=0}^{N-1} e^{-j 2 \pi\left(\nu^{\prime}-\nu_{i}\right) c T} \sum_{d=0}^{M-1} e^{j 2 \pi\left(\tau^{\prime}-\tau_{i}\right) d \Delta f} \\
& =\sum_{i=1}^{P} h_{i}^{\prime} \mathcal{G}\left(\nu^{\prime}, \nu_{i}\right) \mathcal{F}\left(\tau^{\prime}, \tau_{i}\right),
\end{aligned}
$$

where $h_{i}^{\prime}=h_{i} e^{-j 2 \pi \nu_{i} \tau_{i}}, \mathcal{F}\left(\tau^{\prime}, \tau_{i}\right)=\sum_{d=0}^{M-1} e^{j 2 \pi\left(\tau^{\prime}-\tau_{i}\right) d \Delta f}$, and $\mathcal{G}\left(\nu^{\prime}, \nu_{i}\right)=\sum_{c=0}^{N-1} e^{-j 2 \pi\left(\nu^{\prime}-\nu_{i}\right) c T}$. Define $\tau_{i}=\frac{\alpha_{i}}{M \Delta f}$ and $\nu_{i}=\frac{\left(\beta_{i}+\gamma_{i}\right)}{N T}$, where $\alpha_{i}$ and $\beta_{i}$ are integers denoting the indices of the delay tap (with delay $\tau_{i}$ ) and Doppler tap (with Doppler value $\nu_{i}$ ), and $0 \leq \gamma_{i}<1$, where $\gamma_{i}$ is called the fractional Doppler which is needed because Doppler shifts are not exactly at the sampling points in the delay-Doppler plane. Now, for calculating $\sqrt{11}, \mathcal{F}\left(\tau^{\prime}, \tau_{i}\right)$ is evaluated at $\tau^{\prime}=\frac{(l-m)}{M \Delta f}$ as

$$
\begin{aligned}
\mathcal{F}\left(\frac{l-m}{M \Delta f}, \tau_{i}\right) & =\sum_{d=0}^{M-1} e^{j \frac{2 \pi}{M}\left(l-m-\alpha_{i}\right) d} \\
& =\frac{e^{j 2 \pi\left(l-m-\alpha_{i}\right)}-1}{e^{j \frac{2 \pi}{M}\left(l-m-\alpha_{i}\right)}-1}
\end{aligned}
$$

which evaluates to $M$ if $\left(l-m-\alpha_{i}\right) \bmod M=0$ and to 0 otherwise . Also,

$$
\mathcal{G}\left(\frac{k-n}{N T}, \nu_{i}\right)=\frac{e^{-j 2 \pi\left(k-n-\beta_{i}-\gamma_{i}\right)}-1}{e^{-j \frac{2 \pi}{N}\left(k-n-\beta_{i}\right)-\gamma_{i}}-1} .
$$

When $\frac{\pi}{N}\left(\left(k-n-\beta_{i}\right)-\gamma_{i}\right)$ is small, we need to consider only $\left(2 E_{i}+1\right)$ significant values of $\mathcal{G}\left(\frac{k-n}{N T}, \nu_{i}\right)$ for $n=\left(k-\beta_{i}+q\right)$ $\bmod N$ and $-E_{i} \leq q \leq E_{i}, E_{i} \ll N$.

Using the above equations, the OTFS input-output relation in (10) for the channel in (15) can be derived as [11]

$$
\begin{aligned}
y[k, l]=\sum_{i=1}^{P} & \sum_{q=-E_{i}}^{E_{i}} h_{i}^{\prime}\left(\frac{e^{-j 2 \pi\left(-q-\gamma_{i}\right)}-1}{N\left(e^{-j \frac{2 \pi}{N}\left(-q-\gamma_{i}\right)}-1\right)}\right) \\
& \times x\left[\left(\left(k-\beta_{i}+q\right)\right)_{N},\left(\left(l-\alpha_{i}\right)\right)_{M}\right]+v[k, l],
\end{aligned}
$$

where $((.))_{N}$ denotes modulo $N$ operation and $v[k, l] \sim$ $\mathcal{C N}\left(0, \sigma^{2}\right)$ is the additive Gaussian noise (AWGN). The fractional Doppler $\gamma_{i}$ affects the neighboring Doppler taps $\left(-E_{i}\right.$ to $\left.E_{i}\right)$ in 19 . This interference is called the inter-Doppler interference (IDI). When the Doppler taps are assumed to be integer multiples $\left(\gamma_{i}=0\right), 19$ simplifies to

$$
\left.y[k, l]=\sum_{i=1}^{P} h_{i}^{\prime} x\left[\left(\left(k-\beta_{i}\right)\right)_{N},\left(\left(l-\alpha_{i}\right)\right)_{M}\right)\right]+v[k, l] .
$$

We use (19) for OTFS signal detection in Sec. IV and 20, for channel estimation in the delay-Doppler domain in Sec. V The equations (19) and (20) can be represented in vectorized form as [11]

$$
\mathbf{y}=\mathbf{H x}+\mathbf{v}
$$

where $\mathbf{x}, \mathbf{y}, \mathbf{v} \in \mathbb{C}^{N M \times 1}, \mathbf{H} \in \mathbb{C}^{N M \times N M}$, the $(k+N l)$ th element of $\mathbf{x}, x_{k+N l}=x[k, l], k=0, \cdots, N-1, l=$ $0, \cdots, M-1$, and the same relation holds for $\mathbf{y}$ and $\mathbf{z}$ as well. In this representation, there are only $P\left(2 E_{i}+1\right)$ nonzero elements in each row and column of $\mathbf{H}$ due to modulo operations.

\section{OTFS Signal DETECTION}

In this section, we present OTFS signal detection algorithms using MCMC sampling based techniques.

\section{A. OTFS signal detection using MCMC sampling}

MCMC techniques are computational techniques commonly used for calculating complex integrals by expressing the integral as an expectation of some probability distribution and then estimating this expectation from the generated samples of that distribution. In this technique, a new sample value is generated randomly from the most recent sample. The transition probabilities between samples are only a function of the previous sample, and hence the name MCMC. MCMC techniques have been used for signal detection in multiuser and MIMO systems [15]-[18]. Gibbs sampling is a well known MCMC technique. Here, we present low-complexity Gibbs sampling based algorithms for OTFS signal detection using the vectorized formulation of the OTFS signal in 21).

1) Gibbs sampling based OTFS detection: Let $\mathbb{A}$ denote the modulation alphabet used (e.g., BPSK, QAM). Assuming that the input symbols are equally likely, the maximum likelihood (ML) decision rule for the signal model in 21 is given by

$$
\hat{\mathbf{x}}_{\mathrm{ML}}=\underset{\mathbf{x} \in \mathbb{A}^{N M}}{\operatorname{argmin}}\|\mathbf{y}-\mathbf{H} \mathbf{x}\|^{2},
$$

which has an exponential complexity in $N M$. However, approximate solutions to 22 can be obtained efficiently using Gibbs sampling based MCMC techniques. The joint probability distribution of interest for detection is

$$
p\left(x_{1}, x_{2}, \cdots, x_{N M} \mid \mathbf{y}, \mathbf{H}\right) \propto \exp \left(-\frac{\|\mathbf{y}-\mathbf{H} \mathbf{x}\|^{2}}{\sigma^{2}}\right) .
$$

Let $t$ denote the iteration index and $k$ denote the coordinate index of $\mathbf{x}$. A random initial vector, denoted by $\mathbf{x}^{(t=0)}$, is chosen for the algorithm to begin. In every iteration, all the $N M$ 
coordinates are updated and the update in the $(t+1)$ th iteration is obtained by sampling from the following distributions:

$$
\begin{aligned}
x_{1}^{(t+1)} & \sim p\left(x_{1} \mid x_{2}^{(t)}, x_{3}^{(t)}, \cdots, x_{N M}^{(t)}, \mathbf{y}, \mathbf{H}\right) \\
x_{2}^{(t+1)} & \sim p\left(x_{2} \mid x_{1}^{(t+1)}, x_{3}^{(t)}, \cdots, x_{N M}^{(t)}, \mathbf{y}, \mathbf{H}\right) \\
x_{3}^{(t+1)} & \sim p\left(x_{3} \mid x_{1}^{(t+1)}, x_{2}^{(t+1)}, x_{4}^{(t)}, \cdots, x_{N M}^{(t)}, \mathbf{y}, \mathbf{H}\right) \\
\vdots & \\
x_{N M}^{(t+1)} & \sim p\left(x_{N M} \mid x_{1}^{(t+1)}, x_{2}^{(t+1)}, \cdots, x_{N M-1}^{(t+1)}, \mathbf{y}, \mathbf{H}\right) .
\end{aligned}
$$

The solution vector thus obtained in the $t$ th iteration is passed to the $(t+1)$ th iteration for the next set of coordinate updates. After a certain number of iterations called the burn-in period, the distribution tends to converge to the stationary distribution [23), which is the distribution to simulate for drawing samples. The detected symbol vector in a given iteration is chosen to be that vector which has the least ML cost $f_{\mathrm{ML}}(\mathbf{x})=\| \mathbf{y}-$ $\mathbf{H x} \|^{2}$ in all the iterations. However, Gibbs sampling algorithm suffers from a problem called stalling, which degrades the BER performance at high SNRs [15]. To alleviate the stalling problem and to reduce the number of iterations, a slightly different distribution can be used for sampling as described in the following subsection.

2) Gibbs sampling with temperature parameter $\alpha$ : The expected number of iterations for finding the solution can be reduced by using a temperature parameter $\alpha$ in the distribution as follows:

$$
p\left(x_{1}, x_{2}, \cdots, x_{N M} \mid \mathbf{y}, \mathbf{H}\right) \propto \exp \left(-\frac{\|\mathbf{y}-\mathbf{H x}\|^{2}}{\alpha^{2} \sigma^{2}}\right) .
$$

However, the value of $\alpha$ to be used is dependent on the operating SNR [17]. Another possible modification of the Gibbs sampling to alleviate the stalling problem is to combine distributions with $\alpha=1$ (conventional) and $\alpha=\infty$ (uniform) using a randomized update rule as given in the next subsection.

3) Randomized Gibbs sampling based detection: Randomized Gibbs sampling algorithm involves a randomization in the update rule. Instead of using the update rule as in conventional Gibbs sampling with probability 1 , a randomized rule using a parameter $r=\frac{1}{N M}$ is used as follows: with probability $(1-r)$, use conventional Gibbs sampling, and with probability $r$, take samples from a uniform distribution. That is, generate $|\mathbb{A}|$ probability values from a uniform distribution given by

$$
p\left(x_{i}^{(t+1)}=i\right) \sim U[0,1], \forall i \in \mathbb{A},
$$

such that $\sum_{i=1}^{|\mathbb{A}|} p\left(x_{i}^{(t+1)}=i\right)=1$, and sample $x_{k}^{(t+1)}$ from this pmf. A listing of the randomized Gibbs sampling based detection algorithm is given in Algorithm 1 .

\section{B. Performance results}

In this subsection, we present the BER performance of OTFS using the randomized Gibbs sampling algorithm based signal detection. Perfect channel knowledge is assumed at the receiver. The number of signal propagation paths (taps) $P$ is taken to be 5. The Doppler model used is given by [11]

$$
\nu_{i}=\nu_{\max } \cos \theta_{i}
$$

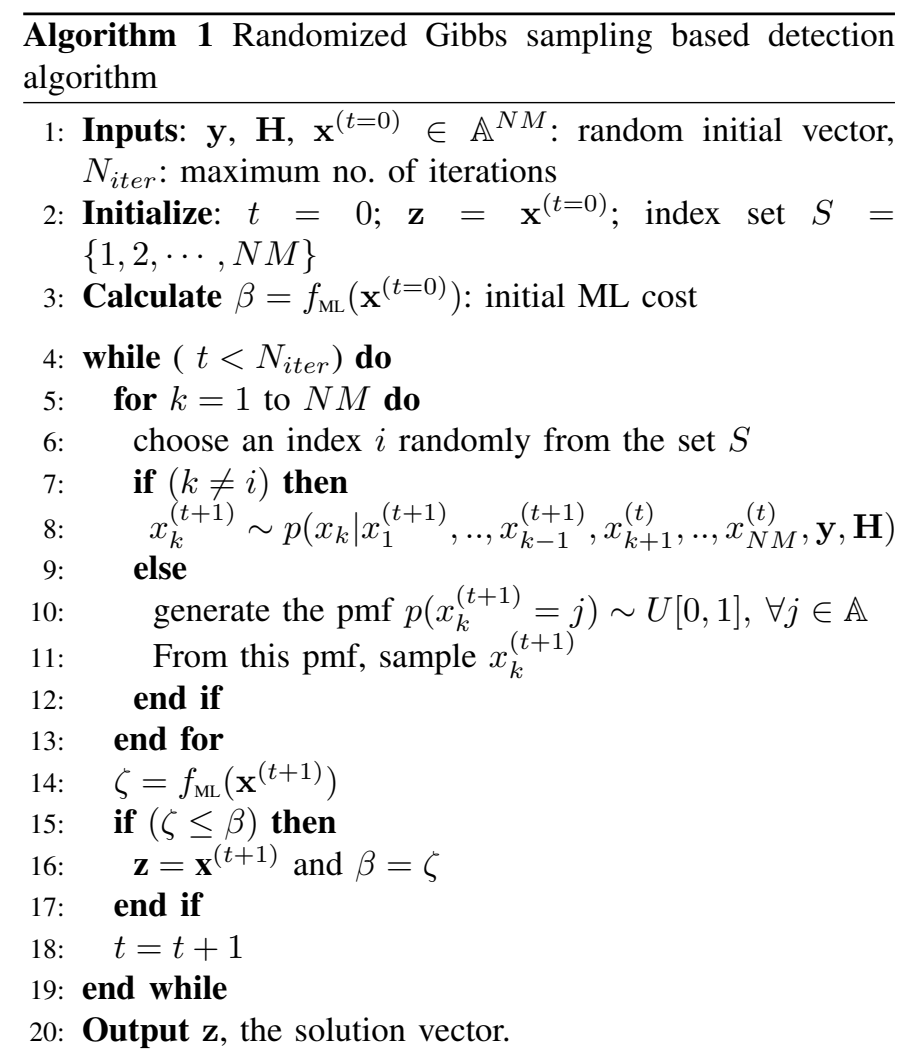

where $\nu_{i}$ is the Doppler shift due to the $i$ th path and $\theta_{i}$ is uniformly distributed, i.e., $\theta_{i} \sim U(0, \pi)$. The 5 -tap delay model in Table I] with $T_{s}=2.1 \mu$ s spacing with a uniform power delay profile is used.

\begin{tabular}{|c|c|c|c|c|c|}
\hline Path index $(i)$ & 1 & 2 & 3 & 4 & 5 \\
\hline Excess Delay $\left(\tau_{i}\right)$ & $0 \mu s$ & $2.1 \mu s$ & $4.2 \mu s$ & $6.3 \mu s$ & $8.4 \mu s$ \\
\hline
\end{tabular}
TABLE I
5AP DELAY MODEL WITH UNIFORM DELAY PROFILE.

The received signal model in $(19)$ is used for the simulation with the parameter $E_{i}=4$. Three different user equipment (UE) speeds, viz., $27 \mathrm{kmph}, 100 \mathrm{kmph}$, and $500 \mathrm{kmph}$ are considered. At a carrier frequency of $4 \mathrm{GHz}$, these speeds correspond to Doppler frequencies $100 \mathrm{~Hz}, 444.44 \mathrm{~Hz}$, and $1851 \mathrm{~Hz}$, respectively. Other simulation parameters used are given in Table II

Figure 3 shows the BER performance of OTFS with $M=$ $128, N=32$, BPSK, $\Delta f=3.75 \mathrm{kHz}$ subcarrier spacing, and a frame length of $M N T_{s}=128 \times 32 \times 2.1 \times 10^{-6}=8.6 \mathrm{msec}$, using randomized Gibbs sampling based detection algorithm. The number of iterations used is 3. From Fig. 3, we observe that a BER of $10^{-3}$ is achieved at an SNR value of about 13 $\mathrm{dB}$ for all the three Doppler frequencies considered. Note that the Doppler spread for $500 \mathrm{kmph}$ UE speed at $4 \mathrm{GHz}$ carrier frequency is $1.851 \mathrm{kHz}$, which in conventional systems like OFDM would cause severe ICI and performance degradation for the considered subcarrier spacing of $3.75 \mathrm{kHz}$. In fact, OFDM is known to breakdown completely at these high Dopplers. Whereas, the the signal localization achieved by 


\begin{tabular}{|l|l|}
\hline Parameter & Value \\
\hline Carrier frequency $(\mathrm{GHz})$ & 4 \\
\hline Subcarrier spacing $(\mathrm{kHz})$ & 3.75 \\
\hline Frame size $(M, N)$ & $(128,32)$ \\
\hline Modulation scheme & BPSK \\
\hline UE speed $(\mathrm{kmph})$ & $27,120,500$ \\
\hline Channel knowledge & Perfect \\
\hline
\end{tabular}

TABLE II

SIMULATION PARAMETERS.

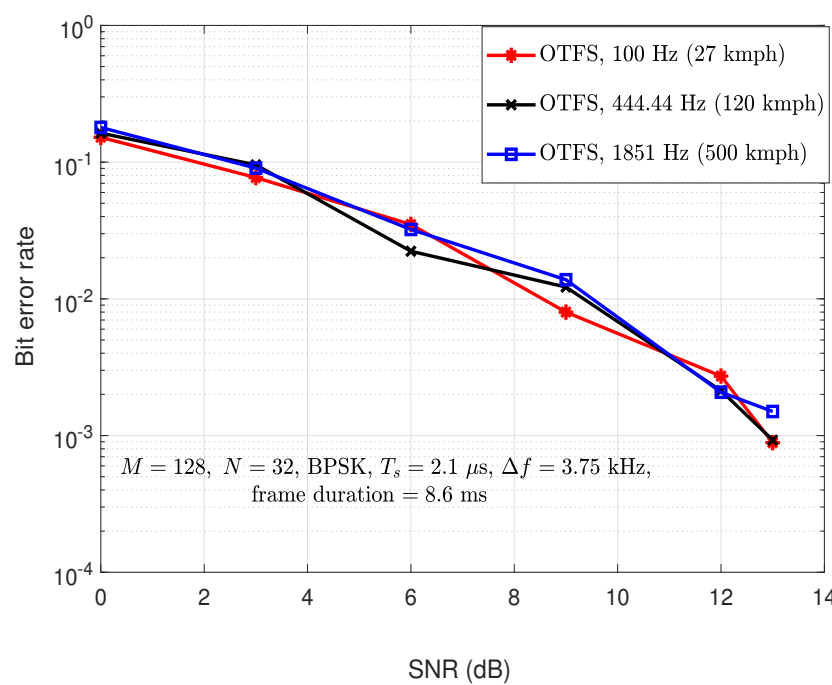

Fig. 3. BER performance of OTFS using randomized Gibbs sampling detection for 3 different Dopplers $100 \mathrm{~Hz}, 444.44 \mathrm{~Hz}$, and $1851 \mathrm{~Hz}$.

OTFS in the delay-Doppler domain renders good BER performance which is almost invariant to Doppler. The BER plots in Fig. 3, therefore, give a clear illustration of the performance robustness of OTFS in high Doppler fading channels.

\section{Delay-Doppler Channel Estimation}

In the previous section, we assumed perfect channel knowledge, i.e., $\mathbf{H}$ is assumed to be perfectly known at the receiver. Here, we relax this assumption and present a method for estimating the channel in the delay-Doppler domain. The method uses a PN sequence as pilot for channel estimation. The estimation approach is as follows. The estimation is done in the discrete domain, where three quantities of interest, namely, delay shift $\left(\delta_{i}\right)$, Doppler shift $\left(\nu_{i}\right)$, and fade coefficient $\left(\alpha_{i}\right)$ for the $i$ th path, for all $i=1, \cdots, P$, need to be estimated. The estimation of $\delta_{i}$ and $\nu_{i}$ is first done by solving a timefrequency shift problem which involves the computation of a matched filter matrix (described later in Sec. V-B1) for each $i$, and the estimates of $\alpha_{i}$ s are obtained to be the values of $P$ highest peaks in the matched filter matrix.

Consider the delay-Doppler channel impulse response $h(\tau, \nu)$ in 15 . The coupling between the input signal and the channel can be written as

$$
y(t)=\sum_{i=1}^{P} h_{i} x\left(t-\tau_{i}\right) e^{j 2 \pi \nu_{i}\left(t-\tau_{i}\right)}+v(t) .
$$

Let $\mathcal{H}$ denote the vector space of complex valued functions on the set of finite integers $\mathbb{Z}_{N_{p}}=\left\{0,1, \cdots, N_{p}-1\right\}$ equipped with addition and multiplication modulo $N_{p}$. The inner product in $\mathcal{H}$ is defined as

$$
\left\langle f_{1}, f_{2}\right\rangle=\sum_{n \in \mathbb{Z}_{N_{p}}} f_{1}[n] f_{2}^{*}[n], \quad f_{1}, f_{2} \in \mathcal{H} .
$$

Also, define $e(t)=e^{\frac{j 2 \pi}{N_{p}} t}$.

\section{A. Channel parameters and the discrete channel model}

Here, we present the discrete channel model for (27), which simplifies the problem of channel estimation (since waveforms becomes sequences). The model in (27) can be written in a slightly modified form as

$$
y(t)=\sum_{i=1}^{P} h_{i}^{\prime} e^{j 2 \pi \nu_{i} t} x\left(t-\tau_{i}\right)+v(t),
$$

where $h_{i}^{\prime}=h_{i} e^{-j 2 \pi \nu_{i} \tau_{i}}, \nu_{i} \in \mathbb{R}, \tau_{i} \in \mathbb{R}_{+}$. The parameters $\left(h_{i}^{\prime}, \nu_{i}, \tau_{i}\right)$ for $i=1,2, \cdots, P$, are called the channel parameters and we have to estimate them. They, in turn, give the estimate of $\mathbf{H}$ in 21). The process for converting the continuous time channel model to a discrete channel model is described as follows [19]. Start with a sequence $S \in \mathcal{H}$ and transmit the following analog signal

$$
S_{A}(t)=\sum_{n=0}^{M-1} S\left[n \bmod N_{p}\right] \operatorname{sinc}(W t-n),
$$

where $M \geq N_{p}$. Let $T_{\text {spread }}=\max \left(\tau_{i}\right)$ denote the time spread of the channel and define $K=\left\lceil W T_{\text {spread }}\right\rceil$. Let $M=N_{p}+K$. Transmit the analog signal $S_{A}(t)$ from time $t=0$ to $t=\frac{M}{W}$. The received signal $R_{A}(t)$ is related to $S_{A}(t)$ through 27). $R_{A}(t)$ is sampled at an interval $T_{s}=\frac{1}{W}$ from time $\frac{K}{W}$, and the sequence $R[n]=R_{A}\left(\frac{(K+n)}{W}\right)$ for $n=0,1, \cdots, N_{p}-1$ is obtained. The following proposition in [19] gives the discrete channel model.

Proposition 1. Let $\tau_{i} \in \frac{1}{W} \mathbb{Z}_{+}$and $\nu_{i} \in \frac{W}{N_{p}} \mathbb{Z}$ for $i=$ $1,2, \cdots, P$. Then $R[n]$ given above satisfies

$$
R[n]=\sum_{i=1}^{P} \alpha_{i} e\left(\omega_{i} n\right) S\left[K+n-\delta_{i}\right]+v[n], \quad n \in \mathbb{Z}_{N_{p}},
$$

where $\alpha_{i}=h_{i}^{\prime} e^{\left(j 2 \pi \nu_{i} K / W\right)}, \delta_{i}=\tau_{i} W$, and $\omega_{i}=N_{p} \nu_{i} / W$.

\section{B. PN pilot based channel estimation}

Let $S, R \in \mathcal{H}$, where $R[n]$ is given by

$$
R[n]=\sum_{i=1}^{P} \alpha_{i} e\left(\omega_{i} n\right) S\left[n-\delta_{i}\right]+v[n], n \in \mathbb{Z}_{N_{p}},
$$

where $\alpha_{i} \in \mathbb{C}, \delta_{i}, \omega_{i} \in \mathbb{Z}_{N_{p}}$, and $v[n] \in \mathcal{H}$. Once $\alpha_{i}, \delta_{i}$, and $\omega_{i}$ are estimated, we can compute $h_{i}^{\prime}, \tau_{i}$, and $\nu_{i}$ for $i=1,2, \cdots, P$ using Proposition 1 , which solves the channel estimation problem. We estimate $\delta_{i}, \omega_{i}$, and $\alpha_{i}$ using a PN pilot based scheme described as follows. 


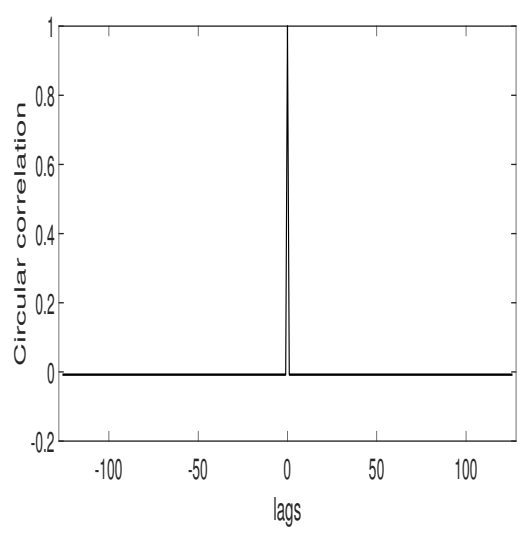

(a) Auto-correlation

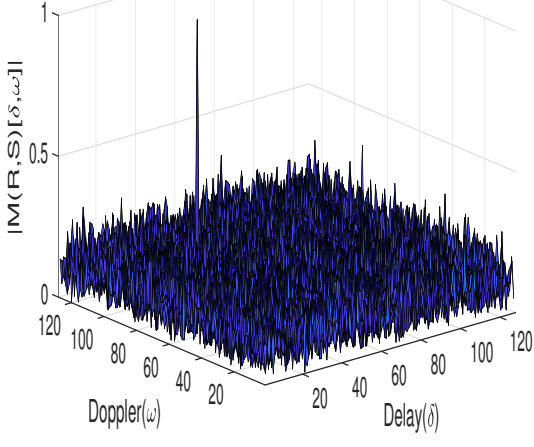

(b) $\left(\delta_{0}, \omega_{0}\right)=(40,90)$

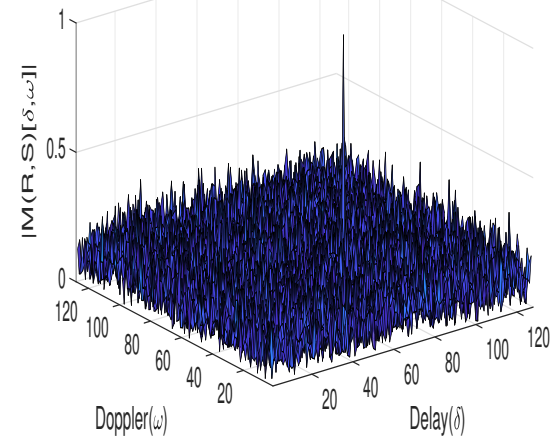

(c) $\left(\delta_{0}, \omega_{0}\right)=(80,60)$

Fig. 4. (a) Auto-correlation function of the PN sequence with $N_{p}=127$. (b), (c) Magnitude of the entries of the matched filter matrix at SNR 0 dB and $N_{p}=127$ for the time-frequency shift problem with $\left(\delta_{0}, \omega_{0}\right)=(40,90)$ and $\left(\delta_{0}, \omega_{0}\right)=(80,60)$, respectively.

1) Solving for $\left(\delta_{i}, \omega_{i}, \alpha_{i}\right)$ : Consider the following simpler variant of (30):

$$
R[n]=e\left(\omega_{0} n\right) S\left[n-\delta_{0}\right]+v[n],
$$

where $n \in \mathbb{Z}_{N_{p}}$ and $\left(\delta_{0}, \omega_{0}\right) \in \mathbb{Z}_{N_{p}} \times \mathbb{Z}_{N_{p}}$. The problem of estimating $\left(\delta_{0}, \omega_{0}\right)$ is called the time-frequency shift problem, which is solved as follows. Define the matched filter matrix of $R$ and $S$ as

$\mathcal{M}(R, S)[\delta, \omega]=\langle R[n], e(\omega n) S[n-\delta]\rangle,(\delta, \omega) \in \mathbb{Z}_{N_{p}} \times \mathbb{Z}_{N_{p}}$.

Suppose $R$ and $S$ satisfy (31) and $S \in \mathcal{H}$ is a PN sequence of norm one and length $N_{p}$. Then, we can obtain the following expression for $\mathcal{M}(R, S)[\delta, \omega]$ by the law of iterated logarithm, with probability going to one, as $N_{p}$ goes to infinity [19]:

$$
\begin{aligned}
\mathcal{M}(R, S)[\delta, \omega] & =1+\epsilon_{N_{p}}^{\prime} \\
& \text { if }(\delta, \omega)=\left(\delta_{0}, \omega_{0}\right) \\
& \epsilon_{N_{p}} \quad \text { if }(\delta, \omega) \neq\left(\delta_{0}, \omega_{0}\right),
\end{aligned}
$$

where $\left|\epsilon_{N_{p}}^{\prime}\right| \leq \frac{1}{\sqrt{N_{p}}}$ and $\left|\epsilon_{N_{p}}\right| \leq \frac{(C+1)}{\sqrt{N_{p}}}$ for some constant $C>0$. Hence, the solution to the time-frequency shift problem is to compute $\mathcal{M}(R, S)$ and choose $\left(\delta_{0}, \omega_{0}\right)$ for which $\mathcal{M}(R, S)\left[\delta_{0}, \omega_{0}\right] \approx 1$. Once $\left(\delta_{i}, \omega_{i}\right)$ 's are obtained as above, the identity (33) along with the bi-linearity of the inner product gives

$$
\alpha_{i} \approx \mathcal{M}(R, S)\left[\delta_{i}, \omega_{i}\right], i=1,2, \cdots, P .
$$

This solves the problem of estimating $\left(\alpha_{i}, \delta_{i}, \omega_{i}\right)$.

Example 1: For illustration, we present plots of the PN sequence ACF and the magnitudes of the entries of the matched filter matrix in Fig. 4. Figure 4(a) shows the ACF of the PN sequence with $N_{p}=127$, and Figs. 4(b) and 4(c) show the magnitudes of the entries of the matched filter matrix for the time-frequency shift problem with $\alpha_{0}=1$ at $\mathrm{SNR}=0 \mathrm{~dB}$ for $\left(\delta_{0}, \omega_{0}\right)=(40,90)$ and $\left(\delta_{0}, \omega_{0}\right)=(80,60)$, respectively. From these figures, we see that the magnitude of the matched filter matrix is close to one when $(\delta, \omega)=\left(\delta_{0}, \omega_{0}\right)$ and is close to zero when $(\delta, \omega) \neq\left(\delta_{0}, \omega_{0}\right)$, as indicated by 33 .
Example 2: The magnitude plots of the entries of the matched filter matrix for channels with $P=2$ at SNR = $20 \mathrm{~dB}$ are shown in Fig. 5(a) and Fig. 5(b), respectively, for $\left(\delta_{0}, \omega_{0}, \delta_{1}, \omega_{1}\right)=(100,30,10,80)$ and $\left(\delta_{0}, \omega_{0}, \delta_{1}, \omega_{1}\right)=$ $(60,20,70,70)$. We can observe that there are two strong peaks at $(\delta, \omega)=\left(\delta_{i}, \omega_{i}\right), i=0,1$. The entries in the matched filter matrix corresponding to these two peaks give the corresponding $\alpha_{i}$ values. The values of the magnitudes are very small when $(\delta, \omega) \neq\left(\delta_{i}, \omega_{i}\right)$.

Example 3: In Fig. 6, we show the effect of $N_{p}$ on the estimation accuracy. This is illustrated using the magnitude plots for a channel with $P=5$ at SNR $=20 \mathrm{~dB}$ and $\left(\delta_{i}, \omega_{i}\right) \in$ $\{(10,60),(20,110),(30,30),(80,40),(110,90)\}$. Two values of $N_{p}$ are considered. Figure 6(a) considers $N_{p}=127$ and Fig. 6(b) considers $N_{p}=1023$. From these two figures, we can see that the magnitudes for $(\delta, \omega) \neq\left(\delta_{i}, \omega_{i}\right)$ for $N_{p}=1023$ are low compared to those for $N_{p}=127$. That is, the estimate can be more accurate for larger values of $N_{p}$, which can be seen from $\sqrt{33}$ and the fact that $\left|\epsilon_{N_{p}}\right|,\left|\epsilon_{N_{p}}^{\prime}\right| \propto 1 / \sqrt{N_{p}}$.

\section{Performance results}

In this subsection, we present the BER performance of OTFS with estimated channel, where the channel parameters (and hence $\mathbf{H}$ ) are estimated using the method described in the previous subsection. The worst case delay and Doppler spread are assumed to be known, so that the parameter $K$ can be chosen a priori in the process of continuous time to discrete time conversion. The channel model in 20 is used, where the delay and Doppler values are assumed to be integer multiples. A carrier frequency of $4 \mathrm{GHz}$ and a channel model with $P=5$ are considered. The delay-Doppler profile considered in the simulation is shown in Table III. A frame size of $(M, N)=(32,32)$, subcarrier spacing of $\Delta f=15$ $\mathrm{kHz}$, uniform power delay profile, and BPSK are considered.

In Fig. 7, we illustrate the accuracy of the proposed channel estimation in terms of the estimation error given by the Frobenius norm of the difference between the channel matrix (H) and the estimated channel matrix $\left(\mathbf{H}_{e}\right)$, i.e., $\left\|\mathbf{H}-\mathbf{H}_{e}\right\|_{F}$. 


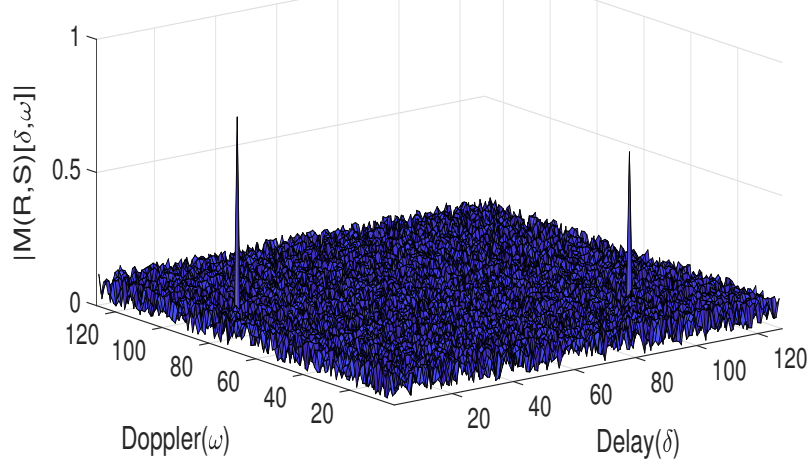

(a) $\left(\delta_{0}, \omega_{0}, \delta_{1}, \omega_{1}\right)=(100,30,10,80)$

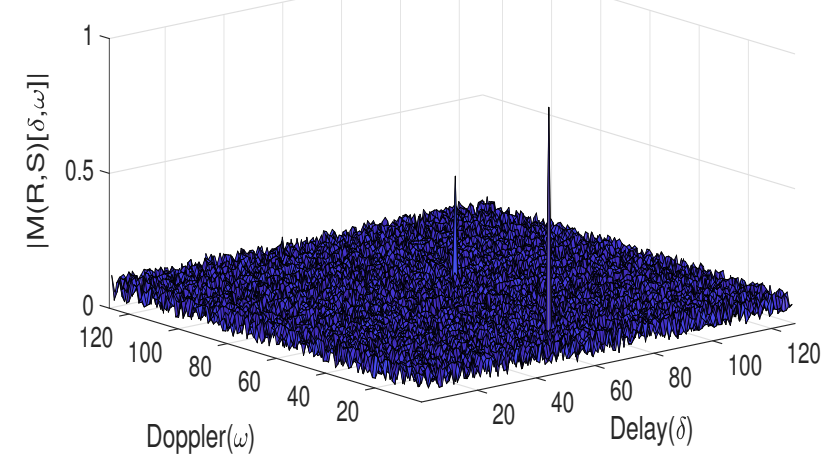

(b) $\left(\delta_{0}, \omega_{0}, \delta_{1}, \omega_{1}\right)=(60,20,70,70)$

Fig. 5. Magnitude of the entries of the matched filter matrix at SNR $=20 \mathrm{~dB}$ and $N_{p}=127$ for channels with $P=2 .\left(\right.$ a) $\left(\delta_{0}, \omega_{0}, \delta_{1}, \omega_{1}\right)=(100,30,10,80)$, (b) $\left(\delta_{0}, \omega_{0}, \delta_{1}, \omega_{1}\right)=(60,20,70,70)$.

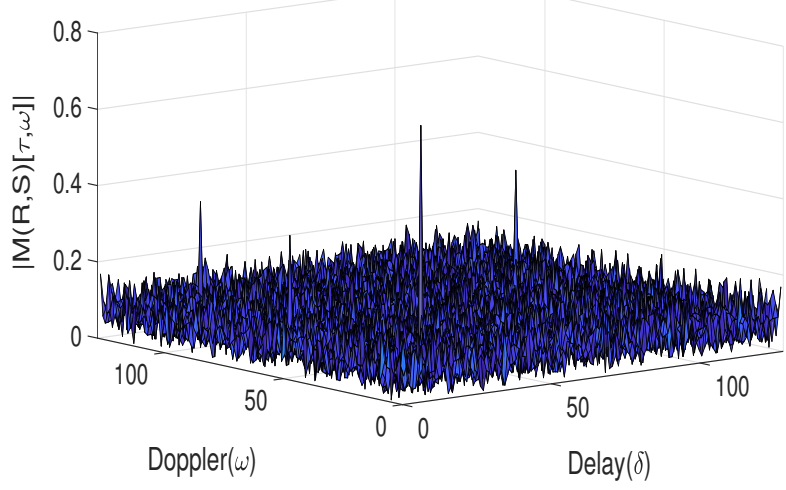

(a) $N_{p}=127$

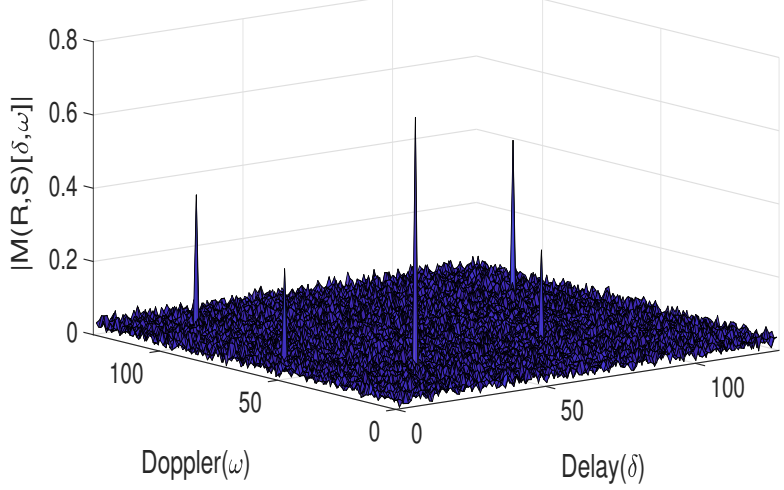

(b) $N_{p}=1023$

Fig. 6. Magnitude of the entries of the matched filter matrix at $\mathrm{SNR}=20 \mathrm{~dB}$ for a channel with $P=5,\left(\delta_{i}, \omega_{i}\right) \in$ $\{(10,60),(20,110),(30,30),(80,40),(110,90)\}$ for (a) $N_{p}=127$ and (b) $N_{p}=1023$.

\begin{tabular}{|c|c|c|c|c|c|}
\hline Path index $(i)$ & 1 & 2 & 3 & 4 & 5 \\
\hline Delay $\left(\tau_{i}\right), \mu \mathrm{s}$ & 2.1 & 4.2 & 6.3 & 8.4 & 10.4 \\
\hline Doppler $\left(\nu_{i}\right), \mathrm{Hz}$ & 0 & 470 & 940 & 1410 & 1880 \\
\hline
\end{tabular}

TABLE III

DELAY-DOPPLER PROFILE FOR THE CHANNEL MODEL WITH $P=5$

Figure 7(a) shows the variation of $\left\|\mathbf{H}-\mathbf{H}_{e}\right\|_{F}$ as a function of pilot SNR for three different values of $N_{p}$ (=31,127,1023). Figure 7(b) shows the variation of $\left\|\mathbf{H}-\mathbf{H}_{e}\right\|_{F}$ as a function of $r$, where $N_{p}=2^{r}-1$, for three different values of SNR $(=0,5,10 \mathrm{~dB})$. From these figures, we see that the estimation error decreases as SNR increases, which is expected. Also, larger PN sequence lengths $\left(N_{p}\right)$ reduce the estimation error because of the $1 / \sqrt{N_{p}}$ relation between $N_{p}$ and the estimation error, as indicated by (33).

In Fig. 8 , we present the BER performance of OTFS with the estimated channel matrix. Detection using Gibbs sampling algorithm with different temperature parameters are consid- ered. Figures $8(\mathrm{a})$ and $8(\mathrm{~b})$ show the BER versus SNR plots for the cases of perfect channel knowledge and estimated channel knowledge. In Fig. 8(a) BER performance when $N_{p}=1023$ and $\alpha=1.5,2$ are shown. In Fig. 8(b), BER performance for $N_{p}=15,127,1023$ when $\alpha=2$ are shown. It is observed that the the performance degradation with estimated channel knowledge (relative to the performance with perfect channel knowledge) is not significant when $N_{p}=1023$, because of the low estimation error achieved with such large $N_{p}$. The degradation, however, becomes significant when $N_{p}$ is reduced. For example, the performance degradation is about $1 \mathrm{~dB}$ at $10^{-2}$ BER when $N_{p}=127$, and the degradation gets severe when $N_{p}=15$. While the estimates become more accurate when $N_{p}$ is increased, the estimation complexity also increases with increasing $N_{p}$. Thus, there is a trade-off between the accuracy of estimation and complexity for the choice of $N_{p}$. 


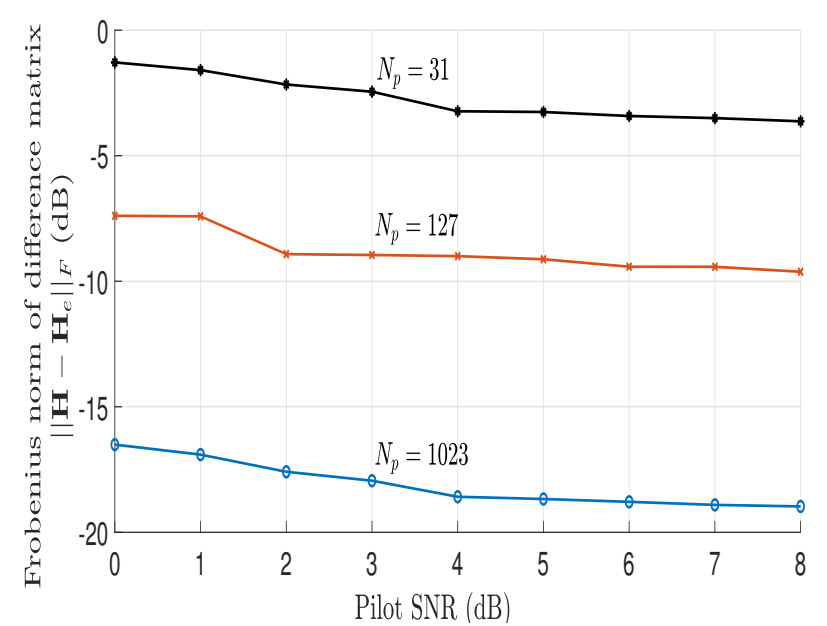

(a)

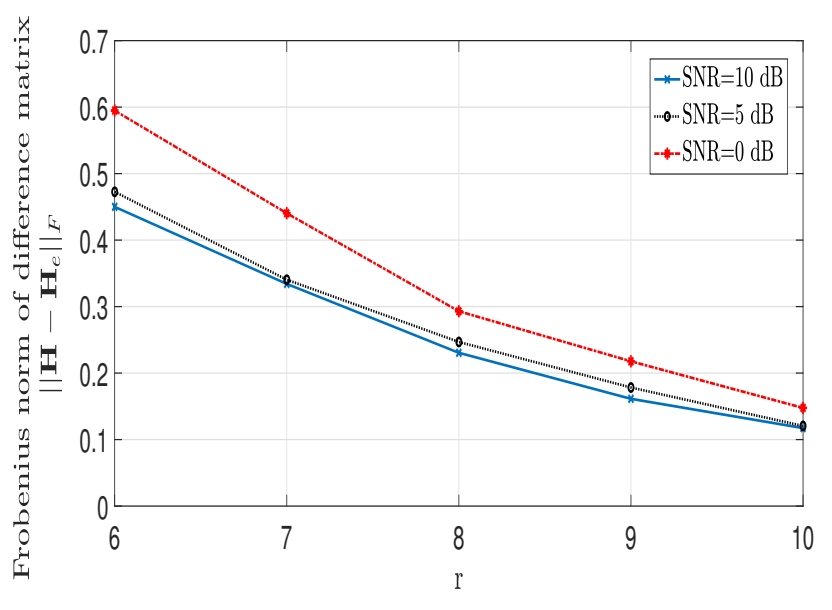

(b)

Fig. 7. Frobenius norm of the difference between the channel matrix $(\mathbf{H})$ and the estimated channel matrix $\left(\mathbf{H}_{e}\right)$ as a function of (a) pilot SNR for various $N_{p}$ values, and (b) $r$, where $N_{p}=2^{r}-1$, for various pilot SNR values.

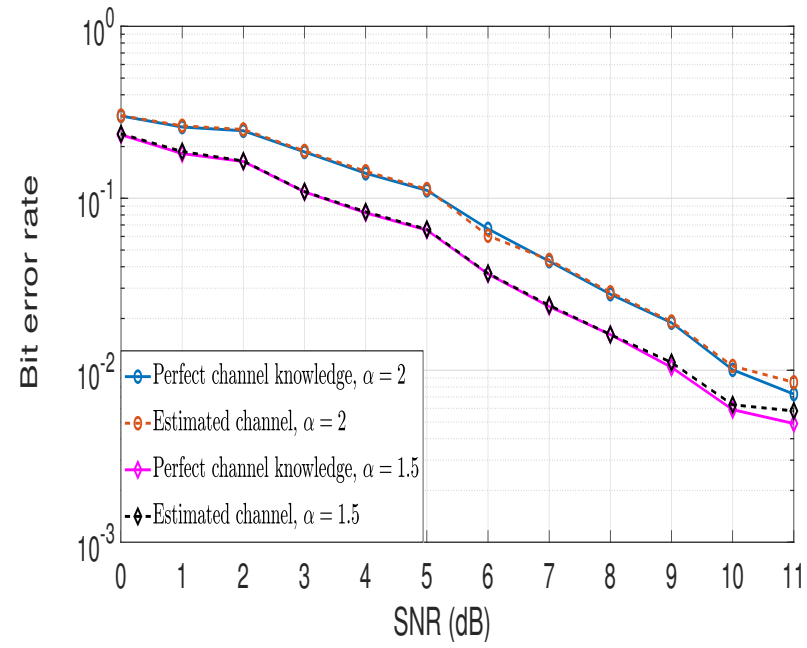

(a)

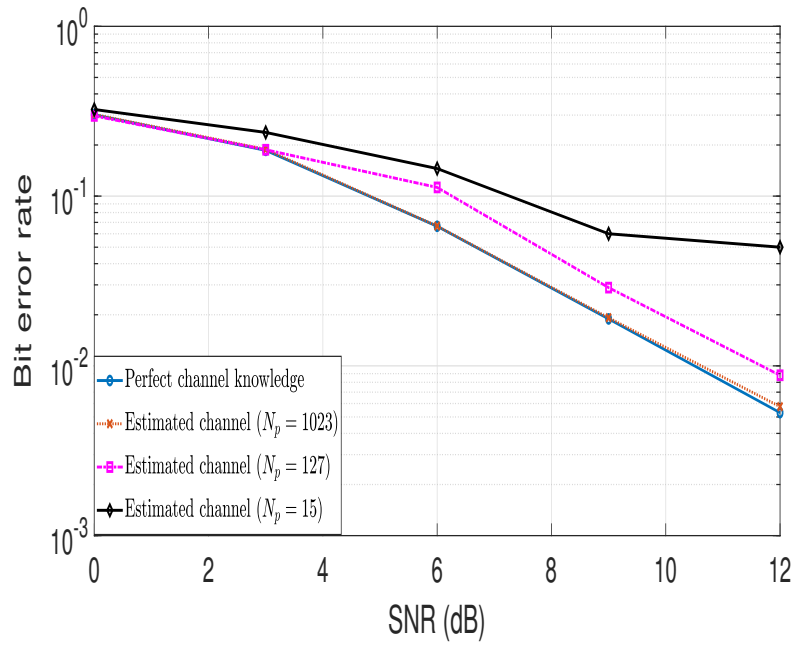

(b)

Fig. 8. BER performance of OTFS with estimated channel. (a) $N_{p}=1023$ and $\alpha=1.5,2$. (b) $\alpha=2$ and $N_{p}=15,127,1023$.

\section{CONCLUSIONS}

We investigated OTFS modulation, which is a recently proposed modulation suited for communication in high-Doppler fading channels, from a signal detection and channel estimation perspective. In particular, we proposed a low-complexity detection scheme based on MCMC sampling techniques and a PN pilot sequence based channel estimation scheme in the delay-Doppler domain. Our results showed that the BER performance of OTFS is robust even at high-Doppler frequencies (e.g., $100 \mathrm{~Hz}, 444 \mathrm{~Hz}$, and $1851 \mathrm{~Hz}$ Dopplers). The proposed channel estimation scheme was shown to achieve small estimation errors and BER degradation for large pilot PN sequence lengths. The feasibility of such simple channel estimation schemes that exploit the fade invariance in the delay-Doppler domain and the robust detection performance even at high Dopplers (a feature that current multicarrier modulation schemes such as OFDM do not offer) suggest that OTFS is a promising next generation modulation scheme suited for $5 \mathrm{G}$ and future wireless systems.

\section{REFERENCES}

[1] W. C. Jakes, Microwave Mobile Communications, New York: IEEE Press, reprinted, 1994.

[2] A. Goldsmith, Wireless Communications, Cambridge Univ. press, 2005.

[3] T. Wang, J. G. Proakis, E. Masry, and J. R. Zeidler, "Performance degradation of OFDM systems due to Doppler spreading," IEEE Trans. Wireless Commun., vol. 5, no. 6, pp. 1422-1432, Jun. 2006.

[4] T. Strohmer and S. Beaver, "Optimal OFDM design for time-frequency dispersive channels," IEEE Trans. Commun., vol. 51, no. 7, pp. 11111122, Jul. 2003.

[5] F-M. Han and X-D. Zhang, "Hexagonal multicarrier modulation: a robust transmission scheme for time-frequency dispersive channels," IEEE Trans. Signal Process., vol. 55, no. 5, pp. 1955-1961, May 2007. 
[6] F-M. Han and X-D. Zhang, "Wireless multicarrier digital transmission via Weyl-Heisenberg frames over time-frequency dispersive channels," IEEE Trans. Commun., vol. 57, no. 6, pp. 1721-1733, Jun. 2009.

[7] A. Monk, R. Hadani, M. Tsatsanis, and S. Rakib, "OTFS - orthogonal time frequency space: a novel modulation technique meeting $5 \mathrm{G}$ high mobility and massive MIMO challenges," online: arXiv:1608.02993 [cs.IT] 9 Aug 2016.

[8] R. Hadani, S. Rakib, M. Tsatsanis, A. Monk, A. J. Goldsmith, A. F. Molisch, and R. Calderbank, "Orthogonal time frequency space modulation," Proc. IEEE WCNC'2017, pp. 1-7, Mar. 2017.

[9] R. Hadani, S. Rakib, A. F. Molisch, C. Ibars, A. Monk, M. Tsatsanis, J Delfeld, A. Goldsmith, and R. Calderbank, "Orthogonal time frequency space (OTFS) modulation for millimeter-wave communications systems," in Proc. IEEE MTT-S Intl. Microwave Symp., pp. 681-683, Jun. 2017.

[10] L. Li, H. Wei, Y. Huang, Y. Yao, W. Ling, G. Chen, P. Li, and Y. Cai, "A simple two-stage equalizer with simplified orthogonal time frequency space modulation over rapidly time-varying channels," online: arXiv:1709.02505 1 [cs.IT] 8 Sep 2017.

[11] P. Raviteja, K. T. Phan, Q. Jin, Y. Hong, and E. Viterbo, "Lowcomplexity iterative detection for orthogonal time frequency space modulation," online: arXiv:1709.09402 v1 [cs.IT] 27 Sep 2017.

[12] A. Farhang, A. Rezazadeh Reyhani, L. E. Doyle, and B. FarhangBoroujeny, "Low complexity modem structure for OFDM-based orthogonal time frequency space modulation," IEEE Wireless Commun. Lett., doi: 10.1109/LWC.2017.2776942, Nov. 2017.

[13] A. Rezazadeh Reyhani, A. Farhang, M. Ji, R-R. Chen, and B. FarhangBoroujeny, "Analysis of discrete-time MIMO OFDM-based orthogonal time frequency space modulation," arXiv:1710.07900 1 [cs.IT] 22 Oct 2017.

[14] T. Dean, M. Chowdhury, and A. Goldsmith, "A new modulation technique for Doppler compensation in frequency-dispersive channels," Proc. IEEE PIMRC'2017, Oct. 2017.

[15] B. Farhang-Boroujeny, H. Zhu, and Z. Shi, "Markov chain Monte Carlo algorithms for CDMA and MIMO communication systems," IEEE Trans. Signal Process., vol. 54, no. 5, pp. 1896-1909, May 2006.

[16] R. Peng, R-R. Chen, and B. Farhang-Boroujeny, "Markov chain Monte Carlo detectors for channels with intersymbol interference," IEEE Trans. Signal Process., vol. 58, no. 4, pp. 2206-2217, Apr. 2010.

[17] M. Hansen, B. Hassibi, A. G. Dimakis, and W. Xu, "Near-optimal detection in MIMO systems using Gibbs sampling," in Proc. IEEE GLOBECOM'2009, Dec. 2009, pp. 1-6.

[18] T. Datta, N. Ashok Kumar, A. Chockalingam, and B. Sundar Rajan, "A novel Monte-Carlo-sampling-based receiver for large-scale uplink multiuser MIMO systems," IEEE Trans. Veh. Tech., vol. 62, no. 7, pp. 3019-3038, Sep. 2013.

[19] A. Fish, S. Gurevich, R. Hadani, A. M. Sayeed, and O. Schwartz, "Delay-Doppler channel estimation in almost linear complexity," IEEE Trans. Inf. Theory, vol. 59, no. 11, pp. 7632-7644, Nov. 2013. 